\title{
HELIOSPHERIC IMAGES OF THE SOLAR WIND AT EARTH
}

\author{
N. R. Sheeley, Jr., A. D. Herbst, ${ }^{1}$ C. A. Palatchi, ${ }^{2}$ Y.-M. Wang, R. A. Howard, J. D. Moses, \\ A. Vourlidas, J. S. Newmark, D. G. Socker, S. P. Plunkett, and C. M. Korendyke \\ Space Science Division, Naval Research Laboratory, Washington, DC 20375-5352; \\ sheeley@spruce.nrl.navy.mil \\ L. F. Burlaga, J. M. Davila, W. T. Thompson, and O. C. St Cyr \\ NASA Goddard Space Flight Center, Greenbelt, MD 20742 \\ R. A. Harrison and C. J. Davis \\ Rutherford Appleton Laboratory, Chilton, Didcot, Oxfordshire OX11 OQX, UK \\ C. J. $\mathrm{EyLES}^{3}$ \\ School of Physics and Astronomy, University of Birmingham, Edgbaston, B15 2TT, UK \\ J. P. Halain \\ Centre Spatiale de Liege, University of Liege, Avenue du Pre-Aily, B-4031 Angleur, Belgium \\ D. Wang, N. B. Rich, K. Battams, and E. Esfandiari \\ Interferometrics, Inc., 13454 Sunrise Valley Drive, Herndon, VA 20171 \\ AND \\ G. STEnBorg \\ The Catholic University of America, 620 Michigan Avenue NE, Washington, DC 20064 \\ Received 2007 October 2; accepted 2007 November 9
}

\begin{abstract}
During relatively quiet solar conditions throughout the spring and summer of 2007, the SECCHI HI2 white-light telescope on the STEREO B solar-orbiting spacecraft observed a succession of wave fronts sweeping past Earth. We have compared these heliospheric images with in situ plasma and magnetic field measurements obtained by nearEarth spacecraft, and we have found a near perfect association between the occurrence of these waves and the arrival of density enhancements at the leading edges of high-speed solar wind streams. Virtually all of the strong corotating interaction regions are accompanied by large-scale waves, and the low-density regions between them lack such waves. Because the Sun was dominated by long-lived coronal holes and recurrent solar wind streams during this interval, there is little doubt that we have been observing the compression regions that are formed at low latitude as solar rotation causes the high-speed wind from coronal holes to run into lower speed wind ahead of it.
\end{abstract}

Subject headings: Sun: corona - Sun: coronal mass ejections (CMEs) - Sun: magnetic fields

\section{INTRODUCTION}

The Solar Terrestrial Relations Observatory (STEREO) spacecraft were launched on 2006 October 25 with the objective of obtaining stereoscopic observations of the Sun from a near-Earth orbit. After some initial maneuvers, which included a gravitational assist from the Moon, the two spacecraft achieved their orbits, with one spacecraft (A) located slightly closer to the Sun and gradually moving ahead of Earth, and the other spacecraft (B) located slightly farther from the Sun and gradually falling behind. The angular distance between the A and B spacecraft increases at a rate of approximately $45^{\circ}$ per year and was about $26^{\circ}$ in early September 2007.

Each spacecraft is equipped with a suite of Sun-Earth Connection Coronal and Heliospheric Investigation (SECCHI) instruments (Howard 2007). In addition to an extreme-ultraviolet imager (EUVI), there are two coronagraphs (COR1 and COR2), and two off-pointed heliospheric imagers (HI1 and HI2). COR1 and COR2 are centered on the Sun and have annular fields of view of $1.3-4.0 R_{\odot}$ and $2-15 R_{\odot}$, respectively. HI1 is pointed $13.2^{\circ}$ away

\footnotetext{
${ }^{1}$ Current address: Cornell University, Ithaca, NY.

2 Current address: California Institute of Technology, Pasadena, CA.

3 Also at Rutherford Appleton Laboratory.
}

from the Sun and shows a $20^{\circ}$ field centered near the ecliptic. HI2 is pointed about $53.4^{\circ}$ from the Sun and shows a $70^{\circ}$ field also centered near the ecliptic. The HI1 and HI2 telescopes on the A spacecraft are pointed east of the Sun, and the corresponding telescopes on the B spacecraft are pointed west of the Sun. Together, these telescopes observe a field whose dimensions extend outward to $88.4^{\circ}$ from the Sun in the east and west directions. In this paper, we describe observations from the HI2B instrument, which observes the low-latitude region about $18.4^{\circ}-88.4^{\circ}$ west of the Sun.

The orbital maneuvers left the B spacecraft slightly outside of Earth's 1 AU orbit, so that it would fall behind Earth and gradually separate from the A spacecraft located ahead of Earth. This meant that Earth would lie in the HI2B field of view during the early days of the mission when B was still close to Earth. (During the interval May 7-September 14, the separation increased from $2^{\circ}$ to $14^{\circ}$, which caused Earth to move slowly from east to west across the HI2B field of view.) This circumstance was not appreciated during the planning phase of the mission, when a small trapezoidal occulter was designed to block the very bright radiation from Earth, which would eventually lie on the western edge of the field. Consequently, when Earth appeared in the HI2B field of view in April, its very bright image and the optical reflections caused by this bright image were distracting artifacts. 


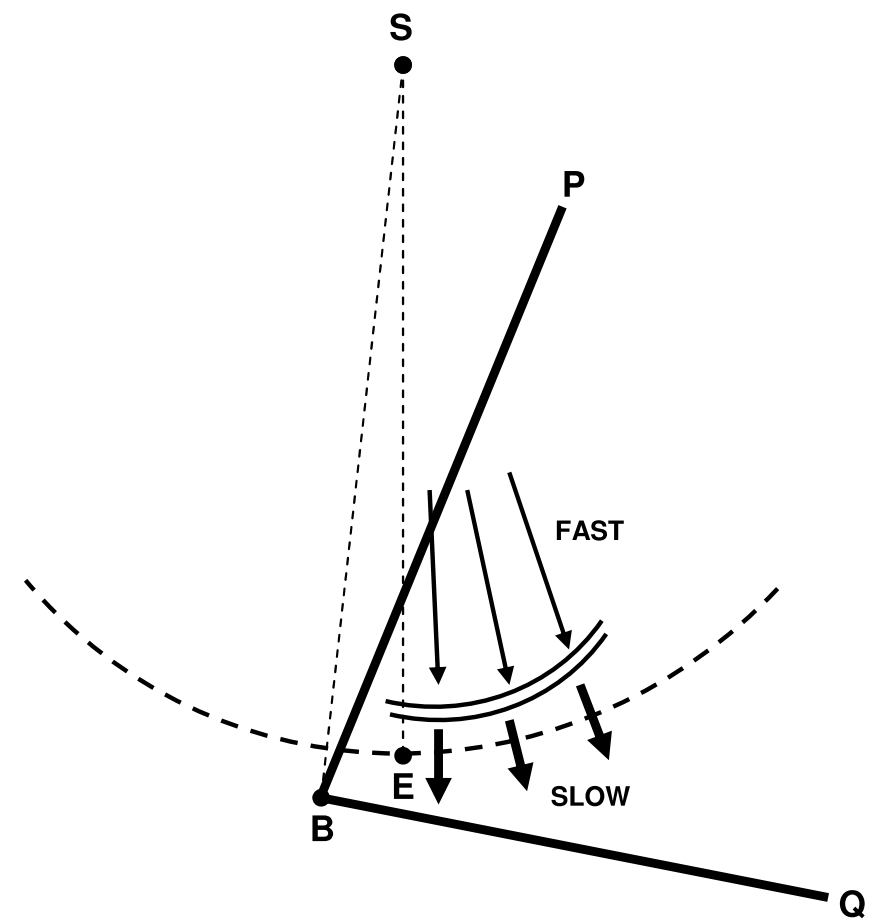

FIG. 1.- Sketch illustrating the passage of a corotating interaction region through the HI2B field of view during the summer of 2007. S marks the Sun, B the B spacecraft, E Earth, and the angle PBQ indicates the heliospheric imager's field of view.

However, as we shall explain below, the appearance of the Earth in the HI2B field of view turned out to be a fortunate circumstance. It allowed us to compare direct images of heliospheric waves with in situ observations of corotating solar wind streams at a time that the low-latitude heliosphere was dominated by those streams. In this case, the scientific opportunity greatly outweighed the cosmetic distraction of the Earth and its optical artifacts.

\section{OBSERVATIONS}

Figure 1 illustrates the location of the $\mathrm{B}$ spacecraft relative to the Earth (E), the Sun (S), and the interface between a high-speed stream and the slower wind ahead of it as this interface moves outward toward the Earth. This orbital configuration occurred in July, when the Sun-centered angle BSE was about $8^{\circ}$. The solid lines, bounding the $70^{\circ}$ angle PBQ, indicate the field of view of the HI2B instrument, whose inner edge is located about $20^{\circ}$ from the Sun-B line (i.e., angle $\mathrm{SBP}=20^{\circ}$ ).

The HI2 telescopes provide wideband images in the 4000$10000 \AA$ region of the spectrum. Despite this wide passband, the intensity of the "coronal" signal is so small that these images are dominated by stars, whose limiting magnitudes are around +11 , as well as the so-called dust or Fraunhofer (F) corona. The contribution of the F-corona was reduced by subtracting a monthly minimum intensity from each image. We attempted to reduce the contribution of stars by shifting each image relative to the next one by an amount that corresponds to the apparent motion of the stars through the sky, and then subtracting these shifted images. This procedure worked fairly well, but optical distortions over the relatively wide field of view and finite spatial resolution limited its effectiveness, especially toward the edge of the field. We cleaned up some of the residual light and dark areas associated with each star by applying a median filter to these difference images. Despite this processing, some exceptionally noisy intervals still occur when dense star fields or the Milky Way pass through the field of view.
Figure 2 shows a time sequence of HI2B running difference images when a large wave passed through the field on 2007 July 19-20. (We call this feature a wave because it looks like the apparent bow waves that we have previously observed as streamer ejecta plow outward through slower material in front of them [Sheeley et al. 2007].) Here, north is up and east is to the left. The diameter of the field is about $70^{\circ}$, with the Sun residing about $20^{\circ}$ outside the eastern edge of the field of view. In the upper left panel, an arrow indicates a large wave moving outward from the Sun toward the Earth, which is marked by another arrow just right of center.

These images contain several artifacts, most of which were produced by the extreme brightness of the Earth. The vertical streaks are caused by bleeding during the exposure and readout of the charge coupled device (CCD) detector. The dark (or bright) area that surrounds the Earth in some images is also an artifact of the exposure to such a bright source. A pair of additional artifacts is also visible to the left of the Earth. These artifacts are reflections of Earth and appear on the opposite side of the optical axis of the telescope. As we will see in subsequent figures, while Earth moved left to right across the field, this pair of artifacts moved in the opposite direction, coinciding with it at the center and leaving (or entering) the field on one side when Earth was leaving (or entering) the field on the other side. The trapezoidal feature projecting into the west side of the image is an occulter designed to mask the Earth (and avoid these cosmetic problems) when Earth eventually resides at the western edge of the field. Finally, the coarse structures, especially in the north part of the field, were caused by the additional despiking, smoothing, and contrast enhancement that we used to clean up the figure and make the waves more visible.

As one can see in Figure 2, the wave is a linear feature extending most of the way in a northeast/southwest direction across the HI2B field of view. During this sequence, the wave moved westward across the field and passed Earth (at least as seen along the line of sight) around 10:10 UT on July 20. In these running difference images, the leading-white/trailing-black signature of the wave indicates that it would appear as an enhancement of intensity if we were able to observe it directly on unsubtracted images. Because we are observing Thomson scattered emission, this enhancement of intensity corresponds to an increase in the number of scattering electrons along the line of sight, and therefore probably also to an increase of density. The apparent width of this enhancement is given by the separation between the black and white components and is $\sim 5^{\circ}$. Also, the speed at which the wave crossed the field is $\sim 1.7^{\circ}$ per hour. (As a rough estimate, these waves take about 1 day to move inward from the edge of the field to the center.)

We can deduce two results from these measurements. First, it would take about $3 \mathrm{hr}$ for the wave to pass Earth (or a near-Earth in situ detector). As we shall see below, this is comparable to the temporal widths of the compression regions in front of highspeed solar wind streams. Second, when projected on a sphere of approximately $1 \mathrm{AU}$ radius, this angular rate corresponds to a very high apparent speed of $1300 \mathrm{~km} \mathrm{~s}^{-1}$. This implies that the wave is close to the B spacecraft and therefore far out of the sky plane. As discussed in the Appendix, if the radial speed were in the nominal range of $300-400 \mathrm{~km} \mathrm{~s}^{-1}$, then the direction of motion would be $\sim 72^{\circ}-77^{\circ}$ out of B's sky plane (and therefore aimed almost directly toward Earth), and the wave would be located only 0.2-0.3 AU from the B spacecraft.

Figure 3 provides in situ measurements from the Wind spacecraft, which support these conclusions. Here, a compression region ahead of a high-speed solar wind stream arrives at the Wind spacecraft around 06:00 UT on July 20. The density is enhanced for 

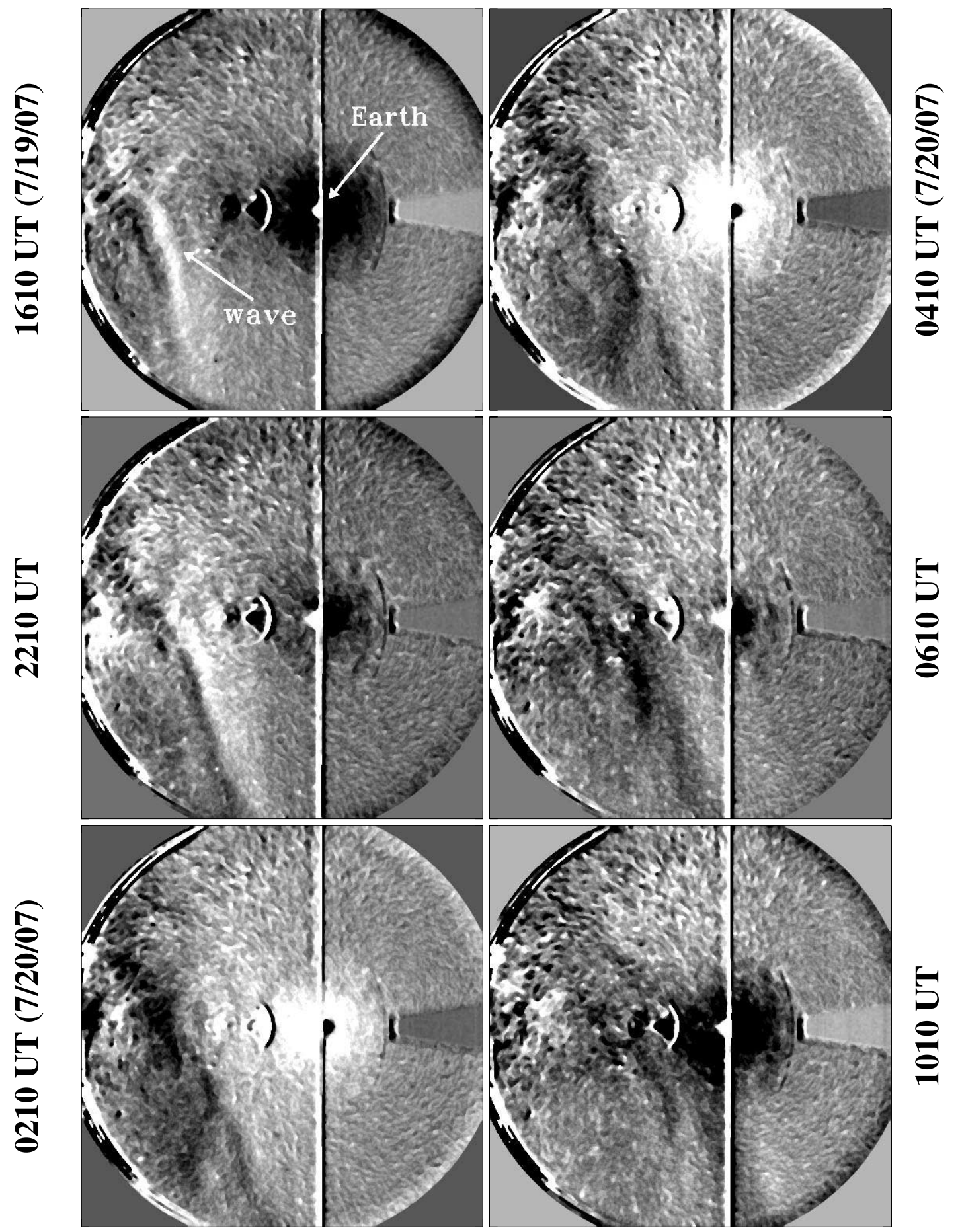

FIG. 2.- Sequence of HI2B running difference images, showing the apparent motion of a large wave left to right past the Earth during 2007 July 19-20. The great brightness of the Earth produced several artifacts, including the vertical streaks of CCD detector bleeding, the sometimes dark and sometimes bright areas around the Earth, and the reflections located across the optical axis on the opposite side of center. The trapezoidal area is an occulter inserted into the field to block the excessive intensity from the Earth when it moves to the right side of the field. The field of view is about $70^{\circ}$ centered $53.4^{\circ}$ west of the Sun. Solar north is up and the Sun is about $18.4^{\circ}$ beyond the left edge of the field.

several hours and reaches its maximum value of $\sim 50$ protons $\mathrm{cm}^{-3}$ around noon. Its full width at half-maximum is $\sim 3-6 \mathrm{hr}$. The sudden drop of density is accompanied by a sudden increase of temperature, a gradual rise in solar wind speed, and a peak in the magnetic field strength. These are classic signatures of a stream interface in front of a high-speed stream from a coronal hole (cf. Burlaga 1995). In this case, the interplanetary field changed its polarity a day earlier on July 19, when the plasma sheet extension of the streamer belt may have been passing the spacecraft.
This association is not a coincidence. It is characteristic of all of the HI2B and in situ observations obtained during 2007 MaySeptember. Figures 4 and 5 provide some of these additional comparisons.

Figure 4 shows the in situ observations during 2007 July 7-28 and provides HI2B images at selected times. Here, the compression regions on July 10, July 20 (already discussed above), and July 26 are all accompanied by HI2B waves. In addition, a small density enhancement on July 13 is accompanied by a very weak 


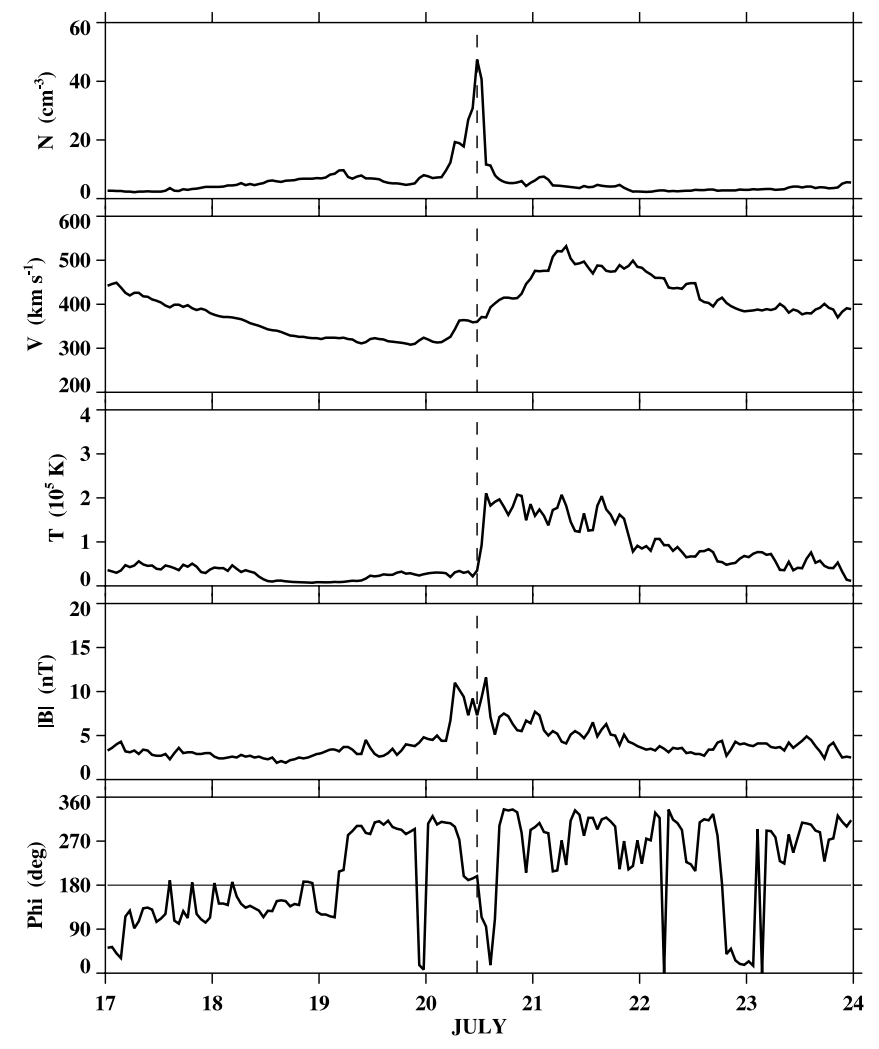

FIG. 3.- Plots of density $(N)$, speed $(V)$, temperature $(T)$, magnetic field strength $(B)$, and azimuthal angle $(\phi)$ from the Wind spacecraft located in a halo orbit at the L1 point about 200 Earth radii in front of the magnetosphere when the HI2B wave shown in Fig. 2 moved past Earth. The arrival of the HI2B wave is accompanied by an in situ density enhancement of about 50 protons $\mathrm{cm}^{-3}$.

extension of a "pseudostreamer" separating coronal holes of the same polarity (Wang et al. 2007). Significantly, the low-density interval on July 17 shows no accompanying wave. The point is that we observe HI2B waves when strong density compressions pass Earth and that we see no waves for the intervening time between those compressions.

Figure 5 shows in situ observations during 2007 May 5-21 and again provides selected HI2B images. In this earlier time interval, the Earth is on the east side of center and its optical reflections are on the west side, which is the opposite of what we observed in July. During May 6-7 a group of waves moves into the HI2B field, merging and passing the Earth around 12:11 UT on May 7, at the time of another classic interaction region ahead of a high-speed stream. The magnetic field's azimuthal angle $\phi$ changed abruptly at this time, indicating the passage of a sector boundary. This is the same sector boundary shown in Figure 4 for the July 26 event.

The image on May 12 is included to again show that no wave is visible during the quiet time between corotating streams. The May 15 image shows a small density wave, which seems to be associated with a small in situ density enhancement in the Wind plasma data. The in situ measurements may indicate a small stream or the edge of a stream that grazed the Wind spacecraft. However, they do not provide a clear example of a corotating interaction region. Finally, the wave on May 18 corresponds to another clear corotating stream. In fact, it is the predecessor of the July 10 stream shown in Figure 4.

The apparent motion of these waves through the HI2B field of view is consistent with a constant radial speed directed far out of the sky plane in the general direction of Earth. This can be appreciated with the help of elongation/time maps analogous to the height/time maps of motions close to the Sun (Sheeley et al. 1999). Figure 6 shows two of these elongation/time maps for the HI2B observations during 2007 July 18-26. These maps will be described in detail elsewhere. Briefly, they were made in two steps. First, the flat CCD images were transformed to a Sun-centered coordinate system in which the projected radial distance indicates solar elongation. Second, for each position angle of interest, radial strips were extracted from the series of transformed running difference images and stacked in chronological order to give rectangular gray-scale maps of differential intensity, like those shown in Figure 6.

Unlike the elongation/maps made from the HI2A observations, these maps from the HI2B observations required additional processing to reduce the contributions from cosmetic defects. Unwanted horizontal and vertical streaks still remain in the maps of Figure 6. Nevertheless, steeply upward curving tracks are clearly visible on July $19-20$ (especially at a position angle of $250^{\circ}$ ) and on July $25-26$ (especially at $262^{\circ}$ ). These tracks correspond to the waves we have described in Figures 2 and 4, and are similar to the tracks of other waves that were associated with compression regions sweeping past Earth during the summer of 2007. The upper panel of Figure 6 also shows a track during July 21-25, whose slope gradually decreases with time. This track was not associated with an obvious density enhancement in the in situ data at Earth.

In general, it is necessary to use different methods for interpreting elongation/time tracks and conventional height/time tracks. At small elongations, the observed motions are usually close to the Sun and show the true accelerations that occur there. At large elongations, the material has essentially left the solar wind acceleration region and reached its asymptotic speed. Moreover, at this great distance from the Sun, a radial direction of motion no longer lies along the observer-centered "sky sphere" where it would provide a constant angular rate. Even for a constant radial speed, the elongation will not change uniformly with time, and the tracks will be curved. As we shall see, the tracks in Figure 6 all refer to motions of roughly the same speed (300-400 $\mathrm{km} \mathrm{s}^{-1}$ ). The tracks of the waves that passed Earth curve steeply upward, indicating motions far from the sky plane, while the slope of the very long track between them decreases gradually, indicating a trajectory that is closer to the sky plane.

Figure 7 shows measurements of elongation $\alpha$ versus time $t$ for the upward curving track in the lower panel of Figure 6 on July 19-20. We have fit those measurements using equations (A2) and (A3) of the Appendix, which assume a constant speed $v_{r}$ along a radial path that is inclined from the sky plane by an angle $\delta$, and where $t_{0}$ is the effective starting time back at the Sun. As we can see, the fit gives a speed $v_{r} \sim 380 \mathrm{~km} \mathrm{~s}^{-1}$ and an inclination $\delta \sim 73^{\circ}$ out of the sky plane. By comparison, in Figure 8 the measurements for the track of decreasing slope on July 21-25 are fit by a radial speed $v_{r} \sim 320 \mathrm{~km} \mathrm{~s}^{-1}$ and an inclination of only $21^{\circ}$. (Because the trajectory lies close to the sky plane, we were able to track this event back into the HI1B field of view and obtain some additional measurements for the fit.)

\section{SUMMARY AND DISCUSSION}

We have systematically compared HI2B heliospheric images during the interval 2007 May-August with in situ plasma and magnetic field measurements at the Wind spacecraft and found a virtually one-to-one association between the passage of large white-light waves and the arrival of density compressions in front of corotating solar wind streams. In addition, we have analyzed elongation/time plots of the waves and found that their tracks are consistent with motions about $73^{\circ}$ out of the sky plane, as seen from the STEREO B spacecraft. Because the angular 


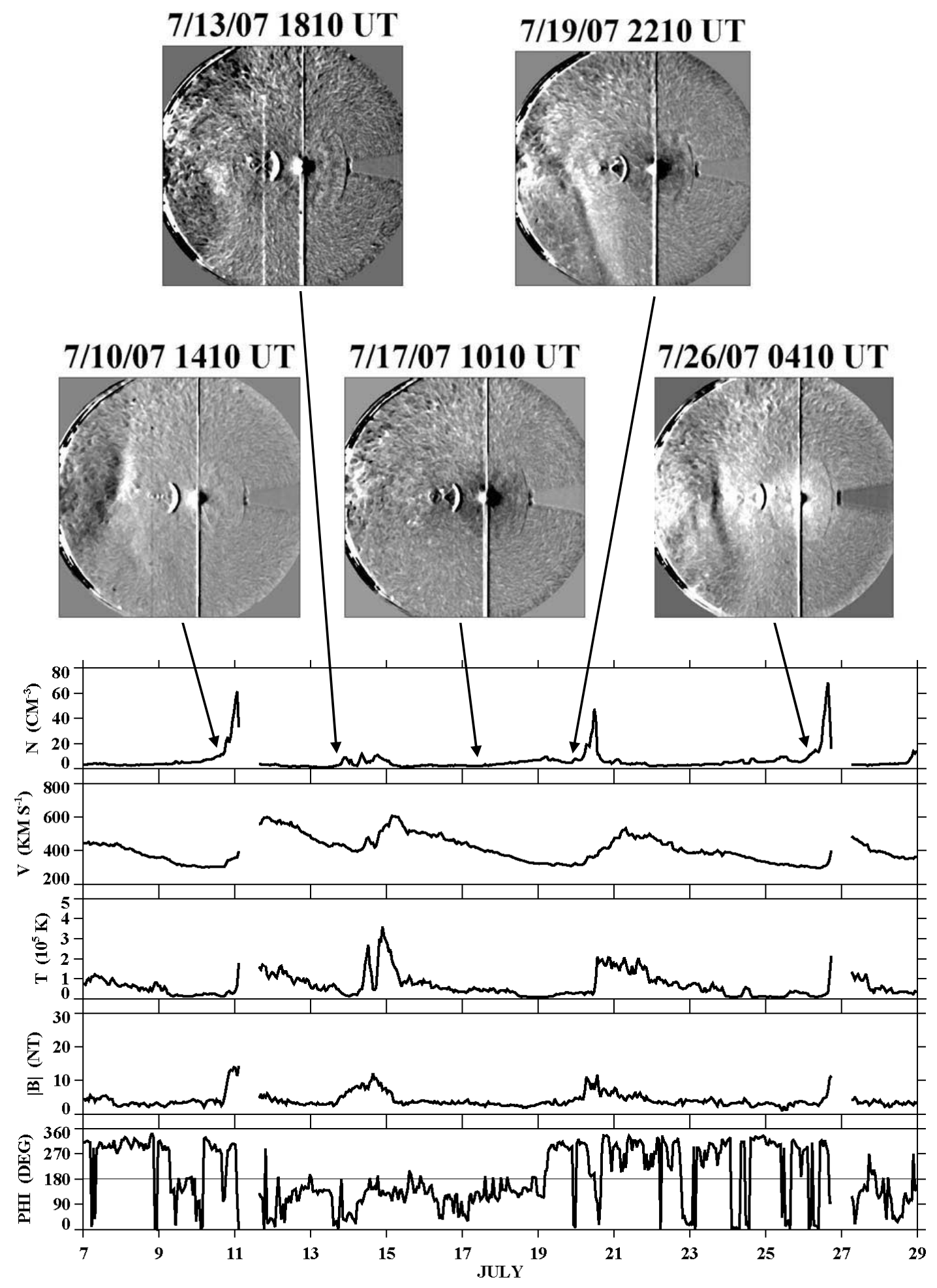

FIG. 4.- Interval of corotating streams and their accompanying HI2B waves during 2007 July $7-28$. The July 13 image was inserted to show that the correlation seems to extend to relatively low density enhancements $\left(\sim 10\right.$ protons $\left.\mathrm{cm}^{-3}\right)$, and the July 17 image was inserted to show that waves are not seen during the low-density intervals in and between the streams. 


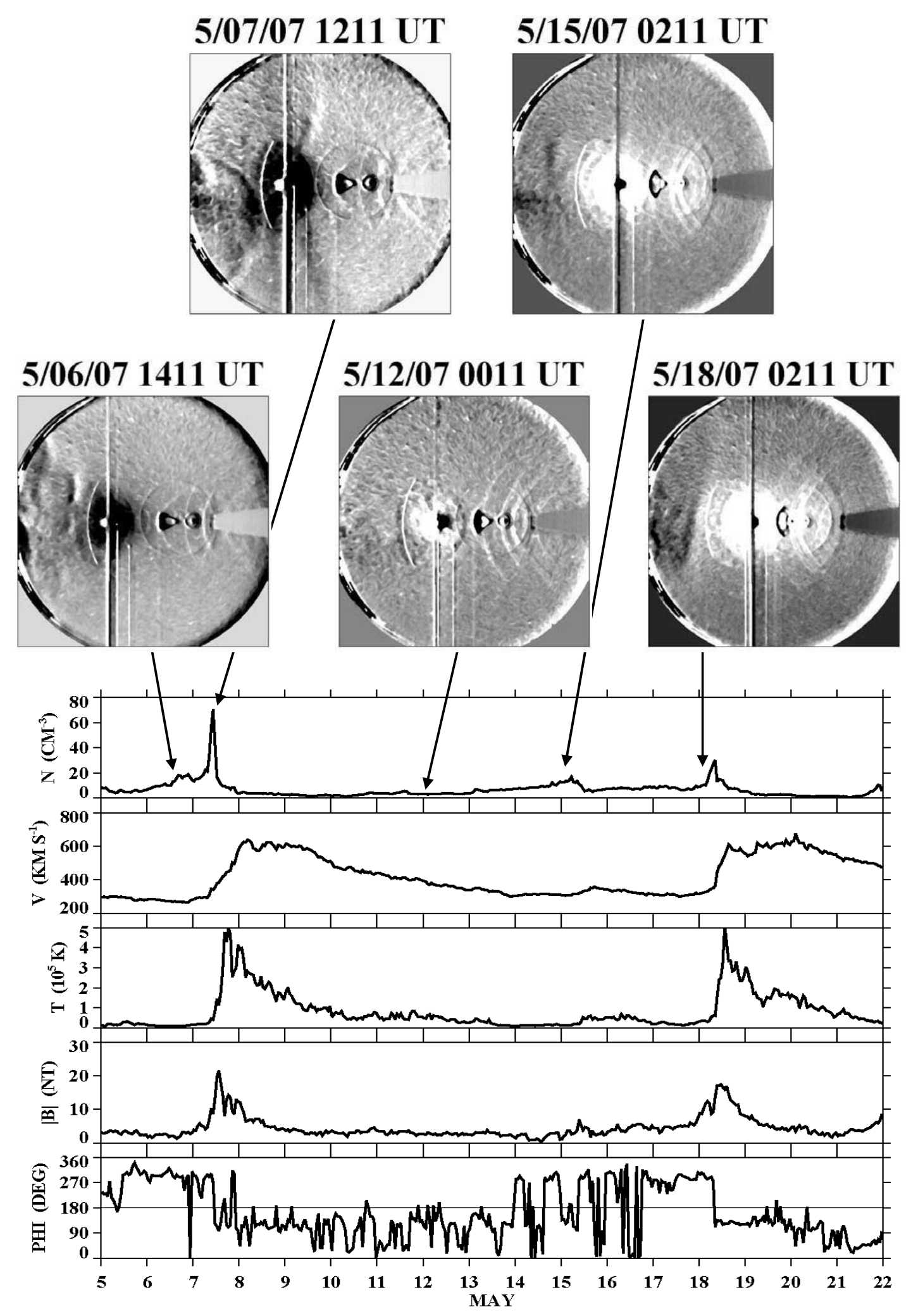

FIG. 5.- A similar comparison during the interval 2007 May 5-21, showing wave-associated density enhancements at the leading edges of major streams on May 6-7

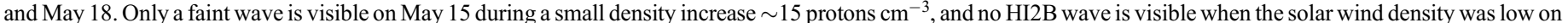
May 12. The May 7 and May 18 events occurred at sector boundaries where the field reverses its direction. 


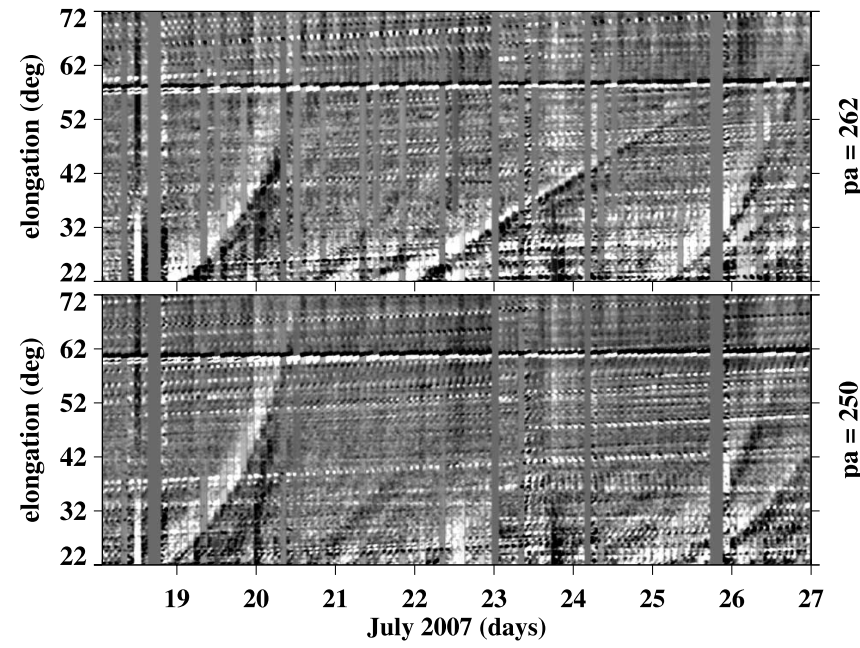

FIG. 6.-Elongation/time maps of HI2B observations at two position angles $\left(250^{\circ}\right.$ and $\left.262^{\circ}\right)$ during 2007 July $18-26$ when two corotating solar wind streams passed through the field of view. Tracks that curve steeply upward toward the location of Earth (near $60^{\circ}$ ) are visible on July 19-20 and July 25-26 in both maps, indicating the broad extent of these nearly head-on events. By comparison, the very long track of declining slope during July $21-25$ is only visible in the map at $262^{\circ}$. Apparently, this event had a smaller angular extent than the other two events and was located much closer to the sky plane, so that it did not pass close to Earth. In each map, the horizontal streaks at $64^{\circ}$ elongation are artifacts indicating where the radial slit used to make these maps intercepts the streaks of CCD detector bleeding (cf. Fig. 2).

separation between $B$ and the Earth was $2^{\circ}-14^{\circ}$ during this time, the waves were even farther out of the sky plane as seen from Earth, and therefore directed within $\sim 15^{\circ}$ of the Sun-Earth line. We suppose that the HI2B instrument is providing images of the density compressions that are driven radially outward by highspeed streams from coronal holes.

For a given elongation angle $\alpha$, the main contribution to the Thomson scattered emission is expected to occur from the region

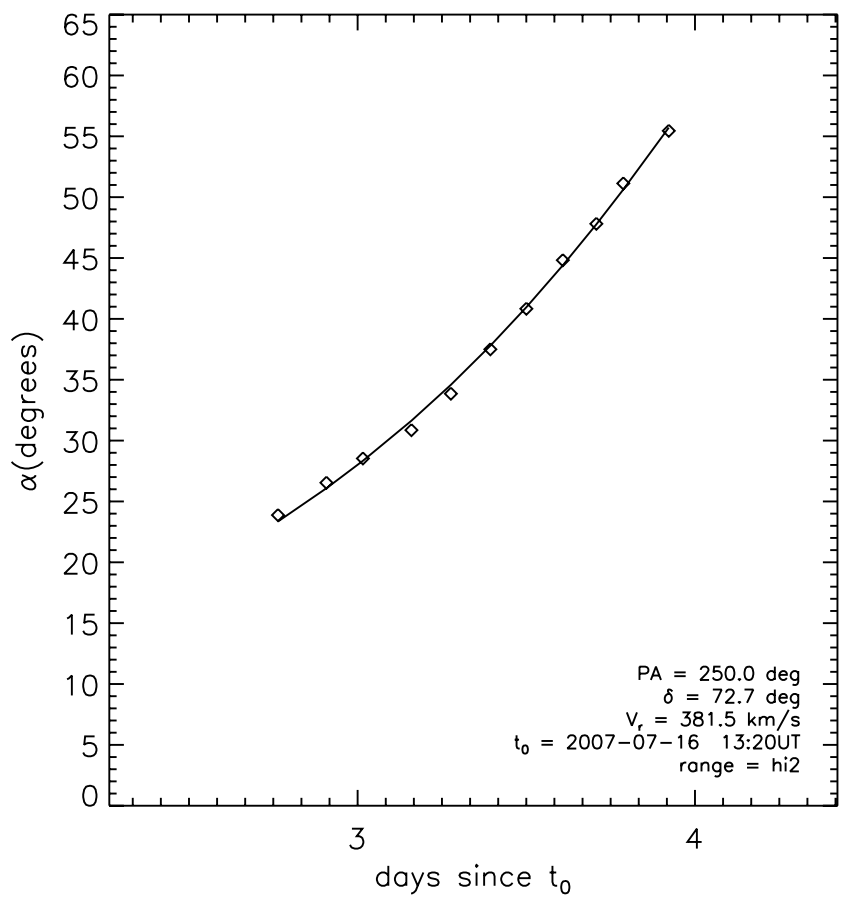

FIG. 7.-Measurements of the elongation/time track on July 19-20 in the lower panel of Fig. 6. The measurements are fit with a curve generated by eqs. (A2)(A3), using a radial speed $\sim 380 \mathrm{~km} \mathrm{~s}^{-1}$ directed $\sim 72^{\circ}$ out of the sky plane.

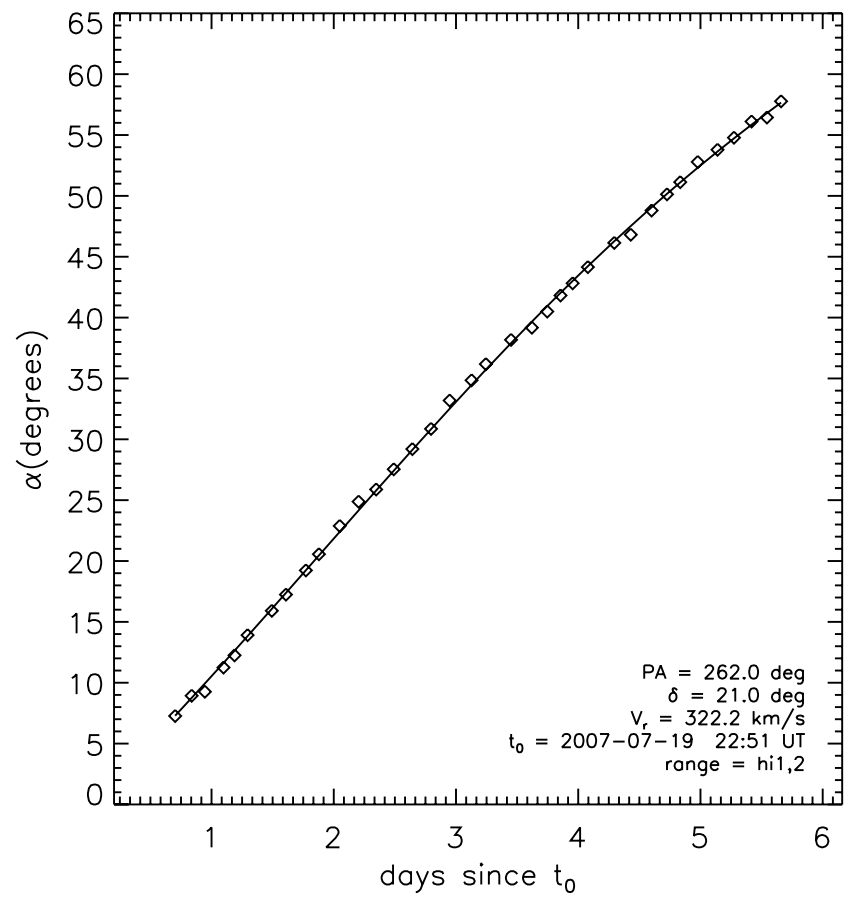

FIG. 8.-Measurements of the elongation/time track on July 21-25 in the upper panel of Fig. 6, again fit using eqs. (A2)-(A3). For this event, the fit was obtained with a radial speed $\sim 320 \mathrm{~km} \mathrm{~s}^{-1}$ directed only $21^{\circ}$ out of the sky plane. Because this motion was relatively close to the sky plane, we were able to track it back into the HI1B field of view and obtain some additional data points for the curve-fitting procedure.

that is closest to the Sun (Vourlidas \& Howard 2006). A similar argument is used to interpret radio scintillation observations of the corona; the main contribution comes from the point on the line of sight that is closest to the Sun, where the density is highest (Bird \& Edenhofer 1990). Referring to Figure 9, this happens when $\alpha=\delta$ and the line of sight BP is perpendicular to the direction of motion SP. According to equation (A6), this is also where the apparent speed $v_{s}$ has its largest value, given by $v_{r} / \cos \alpha$. Thus, the HI2B instrument may be preferentially selecting out segments of the spiral that lie on the so-called Thomson sphere of greatest visibility and whose radial motions have their greatest apparent speed. In contrast, the spiral structure as a whole is not discernible in any given image.

We have not yet performed detailed correlations between the Thomson scattered intensity and the in situ density measurements. However, we found bright waves for in situ densities $\sim 50$ protons $\mathrm{cm}^{-3}$ and faint waves for lower densities $\sim 10-$ 15 protons $\mathrm{cm}^{-3}$. This provides a rough calibration and raises the question of what HI2 may see during more active solar conditions, when shock-associated density increases of $\sim 10-50$ protons $\mathrm{cm}^{-1}$ pass through the field of view.

We are currently attempting to track these HI2 waves backward toward the Sun to see how they originate. We do not expect them to originate in large, fast CMEs, because very few such events occurred during 2007 May-August. Except for a few backside halo CMEs, we are only aware of two fast CMEs, which were visible off the west limb on July 29-30. The slopes of their elongation/time tracks decreased rapidly in the COR2B field of view, indicating a deceleration from $\sim 800$ to about $400 \mathrm{~km} \mathrm{~s}^{-1}$. The tracks flattened out midway through the HI1B field, suggesting a probable location behind the west limb, and never reached the HI2B field.

However, there were several occasions on which material gradually accelerated outward for 1-2 days in the COR2 and LASCO 


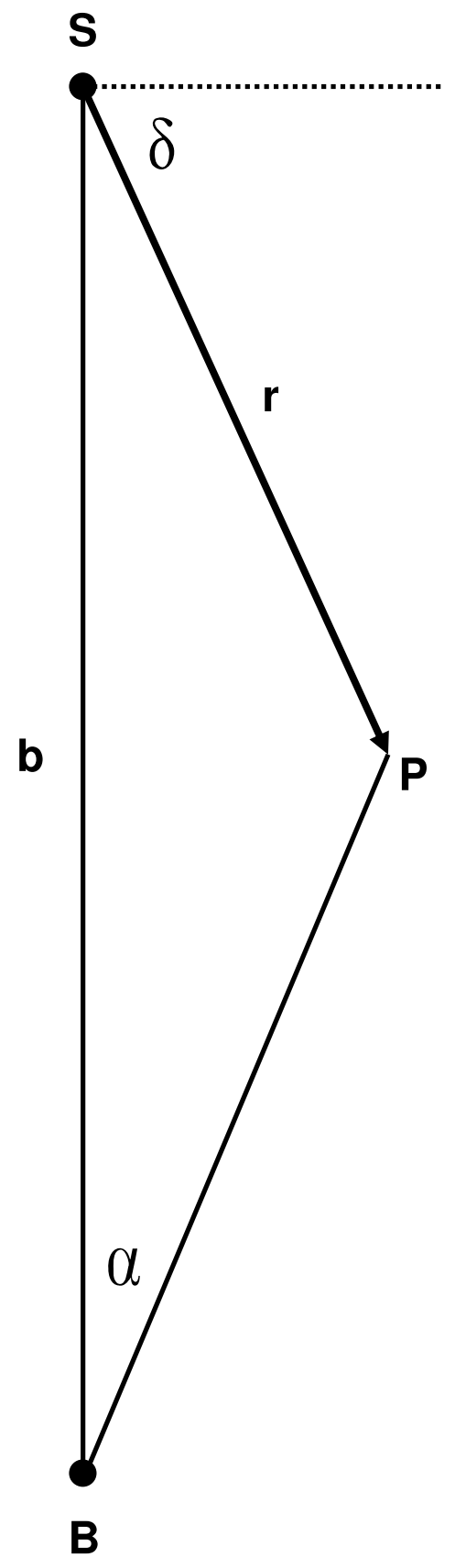

FIG. 9.- Sketch illustrating the geometry of a radially directed ejection $(\mathrm{P})$ relative to the Sun (S) and the B spacecraft (B). $\alpha$ is the elongation angle of the ejection, and $\delta$ is the inclination of the ejection out of the sky plane as seen from B. fields of view before terminating with a streamer detachment or streamer blowout CME. Because these gradually accelerating events generally produce bow waves as their faster components plow forward through the 1-2 day intervals of slower material ahead of them, we were interested to know whether these waves continued outward into the HI2B field of view, perhaps merging to form some of the large corotating density waves. Our preliminary examination of combined COR2-HI1-HI2 elongation/time maps suggests that it is easier to trace features backward through these fields of view if they lie close to the sky plane than if they lie far from it. For example, the July 21-25 track in Figure 6 seems to have originated when the tracks of two or more streamer blobs in the COR2 field on July 19 converged in the HI1 field on July 20-21. Although we have not yet found an origin for the HI2B waves on July 19 and July 26, we think that the May 6 event in Figure 5 originated with a gradual expansion on May 3.

The zodiacal light experiments on the Helios spacecraft and Coriolis satellite have previously detected coronal mass ejections far from the Sun (Leinert et al. 1975; Jackson \& Leinert 1985; Webb et al. 2006). Density enhancements in front of high-speed solar wind streams have also been observed in situ since the flight of Mariner 2 in 1962 (Neugebauer \& Snyder 1962). However, as far as we are aware, the HI2 instruments on the STEREO spacecraft are obtaining the first white-light images of these density enhancements, and in particular, the first images of such solar wind features sweeping past Earth. As the STEREO mission progresses, we expect these observations to provide new knowledge regarding the solar wind and its effects on Earth.

The STEREO/SECCHI data used here are produced by an international consortium of the Naval Research Laboratory (US), Lockheed Martin Solar and Astrophysics Lab (US), NASA Goddard Space Flight Center (US), Rutherford Appleton Laboratory (UK), University of Birmingham (UK), Max-PlanckInstitut für Sonnensystemforschung (Germany), Centre Spatiale de Liege (Belgium), Institut d'Optique Théorique et Appliqueé (France), and the Institut d'Astrophysique Spatiale (France). The US institutions were funded by NASA; the UK institutions by Particle Physics and Astronomy Research Council (PPARC); the German institutions by Deutsches Zentrum für Luft- und Raumfahrt e.V. (DLR); the Belgian institutions by Belgian Science Policy Office; and the French institutions by Centre National d'Etudes Spatiales (CNES) and the Centre National de la Recherche Scientifique (CNRS). The NRL effort was also supported by the USAF Space Test Program, the Office of Naval Research, NASA, and the NRL summer student program.

\section{APPENDIX}

\section{INTERPRETING ELONGATION/TIME MAPS}

In our previous studies of streamer blobs, we found that most of the solar wind acceleration occurs within about $15 R_{\odot}$ of Sun center and that the velocity attains a nearly constant value by the time the material leaves the $30 R_{\odot}$ field of view (Sheeley et al. 1997, 2007). Therefore, we will assume that the flow speed is constant in the HI1 and HI2 fields of view and directed along a radial path inclined by an angle $\delta$ from the sky plane, as indicated in Figure 9. In this figure, $\mathrm{S}$ indicates the Sun, B the B spacecraft, and P the instantaneous location of the moving material. Applying the law of sines to the triangle SBP, we obtain

$$
\frac{r}{b}=\frac{\sin \alpha}{\cos (\alpha-\delta)}
$$



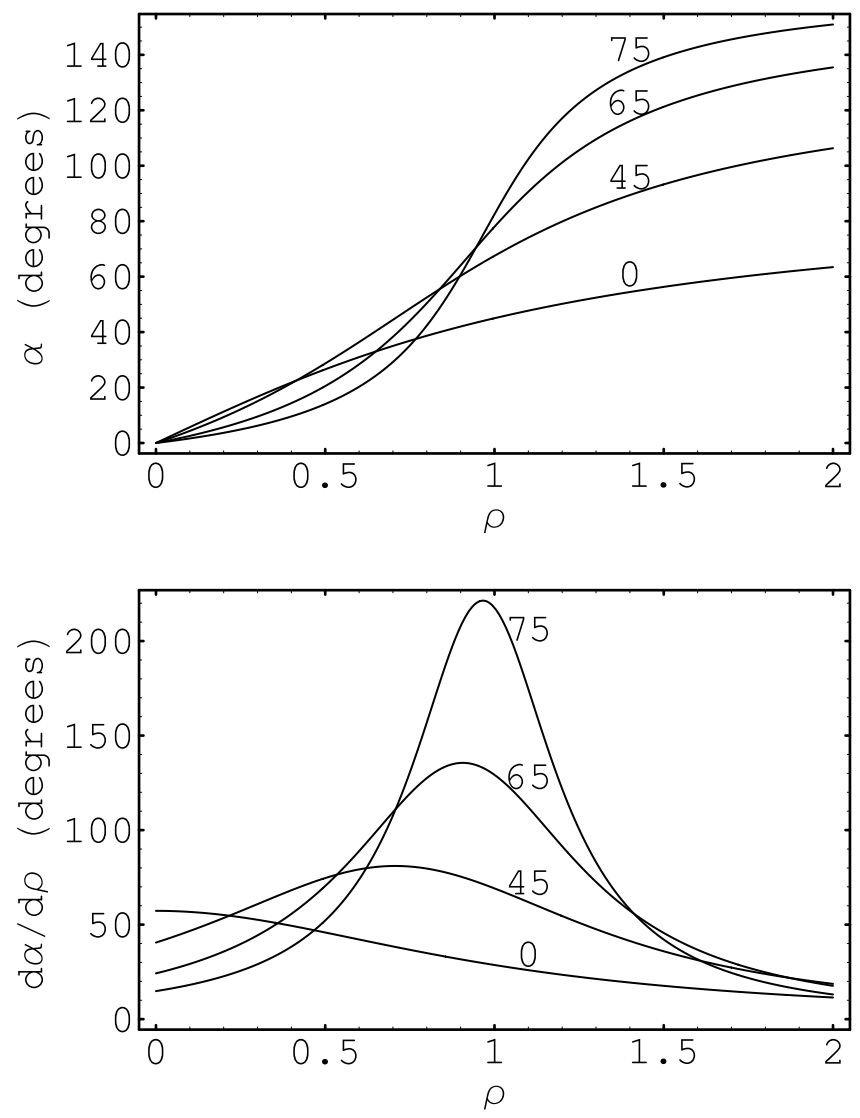

FIG. 10.-Top: Plots of elongation angle $\alpha$ vs. $\rho$ (the radial distance, $r$, in units of the Sun-B distance $b$ ) for values of the sky-plane inclination angle $\delta=0^{\circ}, 45^{\circ}, 65^{\circ}$, and $75^{\circ}$. This panel shows that height/time tracks close to the sky plane begin with a slope corresponding to the true radial speed, and then flatten out, while those far out of the sky plane begin with lower slopes and then become much steeper before eventually flattening out. Bottom: Corresponding plots of the derivative $d \alpha / d \rho$, showing the peaked character of the apparent speed when the trajectories lie far from the sky plane.

where $r$ is the radial distance of the ejection, $b$ is the Sun-B distance (approximately $1 \mathrm{AU}$ ), and $\alpha$ is the elongation angle. One way to proceed is to rewrite this equation with $\alpha$ expressed as a function of the dimensionless parameter $\rho=r / b$,

$$
\tan \alpha=\frac{\rho \cos \delta}{1-\rho \sin \delta} .
$$

If the speed $v_{r}$ is constant, we can regard $\rho$ as a measure of time according to

$$
\rho=\frac{v_{r}\left(t-t_{0}\right)}{b}=\frac{t-t_{0}}{\tau},
$$

where $t_{0}$ is the starting time near the Sun and $\tau$ is the Sun-B transit time $\tau=b / v_{r}$. Thus, together equations (A2) and (A3) provide a relation that can be used to fit elongation/time measurements.

The top panel of Figure 10 shows the elongation angle $\alpha$, plotted as a function of $\rho$ for values of $\delta=0^{\circ}, 45^{\circ}, 65^{\circ}$, and $75^{\circ}$. We can see the different character of these curves for large and small sky-plane inclination angles $\delta$. For motions near the sky plane, the curves begin with a nominal slope (equal to the radial speed $v_{r}$ ) and rapidly flatten out. (The asymptotic angle is $\alpha=\delta+90^{\circ}$.) The effect is even greater for motions behind the sky plane, whose curves would lie below the one shown here for $\delta=0$. However, for motions far from the sky plane and toward the observer, the curves start with lower slopes, corresponding to apparent speeds less than their true radial speeds, and then steepen to values exceeding their radial speeds before leveling out again.

We can evaluate those slopes by taking the derivative of equation (A2) with respect to $\rho$. The result is

$$
\frac{d \alpha}{d \rho}=\frac{\cos \delta}{1-2 \rho \sin \delta+\rho^{2}},
$$

where the slope $d \alpha / d \rho$ is related to the angular speed $d \alpha / d t$ and the apparent speed $v_{s}$ along an idealized sphere of radius $b$ by the equation

$$
v_{r} \frac{d \alpha}{d \rho}=b \frac{d \alpha}{d t}=v_{s} .
$$


The slope $d \alpha / d \rho$ is plotted in the bottom panel of Figure 10. Clearly, the angular speed reaches a peak whose value increases with the inclination $\delta$ of the direction of motion from the sky plane.

Another way to proceed would be to take the derivative of equation (A1) with respect to time. After some rearrangement, the result is

$$
\frac{v_{s}}{v_{r}}=\frac{\cos ^{2}(\alpha-\delta)}{\cos \delta}
$$

where $v_{s}=b d \alpha / d t$ is the apparent speed of the motion along a spherical surface of radius $b$. From equation (A6), we can see that $v_{s}$ obtains its maximum value of $v_{s}=v_{r} / \cos \delta$ when $\alpha=\delta$, and from equation (A4), we can see that it obtains this same value when $\rho=\sin \delta$. Either way, this means that the maximum apparent speed is obtained when the flow moves perpendicular to our line of sight. As we described previously for halo mass ejections (Sheeley et al. 1999), "an object shot nearly at the observer will not show much angular change while it is far away, but will require a rapid neck-wrenching motion to follow when it sails past the observer."

Bird, M. K., \& Edenhofer, P. 1990, Remote Sensing Observations of the Solar Corona (Physics of the Inner Heliosphere I), 13

Burlaga, L. F. 1995, Interplanetary Magnetohydrodynamics (New York : Oxford Univ. Press)

Howard, R. A. 2007, Space Sci. Rev., submitted

Jackson, B. V., \& Leinert, C. 1985, J. Geophys. Res., 90, 10759

Leinert, C., Link, H., Pitz, E., Salm, N., \& Knüppelberg, D. 1975, Raumfahrtforschung, 19,264

\section{REFERENCES}

Neugebauer, M., \& Snyder, C. W. 1962, Science, 138, 1095

Sheeley, N. R., Walters, J. H., Wang, Y.-M., \& Howard, R. A. 1999, J. Geophys. Res., 104, 24739

Sheeley, N. R., Jr., Warren, H. P., \& Wang, Y.-M. 2007, ApJ, 671, 926

Sheeley, N. R., Jr., et al. 1997, ApJ, 484, 472

Vourlidas, A., \& Howard, R. A. 2006, ApJ, 642, 1216

Wang, Y.-M., Sheeley, N. R., Jr., \& Rich, N. B. 2007, ApJ, 658, 1340

Webb, D. F., et al. 2006, J. Geophys. Res. Space Phys., 111, 12101 